\title{
Epidermoid cyst masquerading clinically as hemangioma and radiologically as peripheral nerve sheath tumour. report of a case from a district hospital of Kashmir valley of North India
}

\begin{abstract}
Epidermoid cyst or Sebaceous cyst is a very common lesion in day-to-day dermatological practice. But atypical presentations and unusual locations of these lesions can pose a problem in the diagnosis and management. Here we report the case of a middle aged female who presented with a long duration of epidermoid cyst on face clinically masquerading as hemangioma and radiologically as a peripheral nerve sheath tumour, thus posing a diagnostic and therapeutic dilemma.
\end{abstract}

Keywords: epidermoid cyst, sebaceous cyst, hemangioma, radiologically, peripheral nerve sheath tumour

\author{
Volume 5 Issue I - 2018 \\ Peerzada Sajad,' Sumaya Farooq, ${ }^{2}$ Shaika \\ Qadri, ${ }^{2}$ Sheikh Shahnawaz, ${ }^{3}$ Nazir Shehdad, ${ }^{4}$ \\ Saleem-Ur-Rehman, ${ }^{5}$ GA Ahanger ${ }^{6}$ \\ 'Consultant Dermatologist, Government JLNM Hospital \\ Rainawari, India \\ ${ }^{2}$ Consultant Pathologist, Government JLNM Hospital Rainawari, \\ India \\ ${ }^{3}$ Consultant Radiologist, Government JLNM Hospital Rainawari, \\ India \\ ${ }^{4}$ Medical Superintendant, Government JLNM Hospital Rainawari, \\ India \\ ${ }^{5}$ Director Health Serviices Kashmir, India \\ ${ }^{6}$ Government JLNM Hospital Rainawari, India
}

Correspondence: Peerzada Sajad, Consultant Dermatologist, Government JLNM Hospital Rainawari, 190003, Srinagar, J\&K, India, Tel 9419862252, 7006522775, Email sajads I I2@gmail.com

\section{Clinical presentation}

We hereby report the case of a 48year old normotenive, nondiabetic and euthyroid female who presented with tenyears history of a painless subcutaneous skin coloured to grayish blue swelling of approximately $4 \times 3 \times 2.5 \mathrm{~cm}$ size involving the left preauricular region of face. There was no history of pain, tenderness or local trauma. There was a history of recent increase in size from last 3 months which worried the patient to seek medical consultation. On examination the lesion was slightly mobile, non-tender and adherent to the skin. The lesion was completely blanchable on pressure with a glass slide, thus giving an appearance of hemangioma clinically. There was no punctum on the surface of the lesion (Figure1).

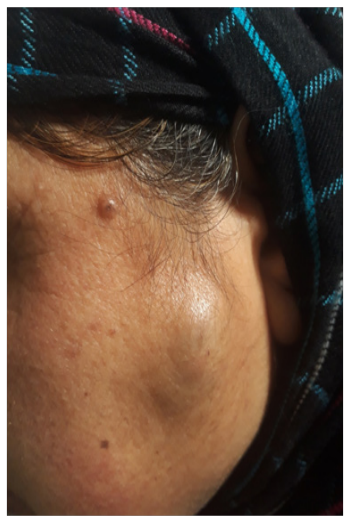

Figure I A middle aged female presenting with an irregular painless subcutaneous skin coloured to grayish blue swelling of approximately $4 \times 3 \times 2.5 \mathrm{~cm}$ size involving the left preauricular region of face.
With a differential diagnosis of hemangioma ,a doppler ultrasonography of the lesion was performed which rather than suggesting a diagnosis of hemangioma complicated the process of differential diagnosis by suggesting a diagnosis of peripheral nerve sheath tumour or a necrotic lymph node, due to the absence of vascularity on colour flow (Figure 2).

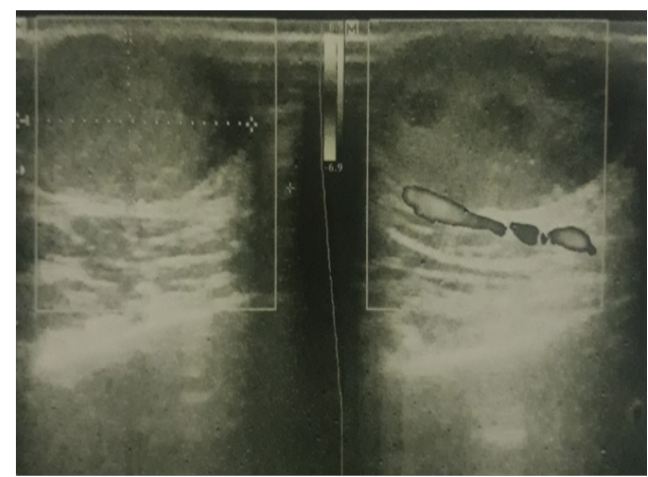

Figure $\mathbf{2}$ Showing a well defined oval encapsulated predominantly hypoechoic lesion seen in subcutaneous location, just anterior to the left parotid gland, overlying the masseter muscle measuring $20.35 \mathrm{~mm} \times 11.02 \mathrm{~mm}$. Note the characteristic absence of vascularity, which hence goes against the diagnosis of hemangioma.

On ultrasonography epidermoid cysts appear as well circumscribed predominantly hypoechoic mass lesions with typical shapes being ovoid to spherical, lobulated and tubular. Small cysts appear as anechoic but larger lesions can appear hetereogenous. Usually there is no associated vascularity, but they can have a variable appearance if ruptured with occasional associated vascularity. The diagnosis of 
peripheral nerve sheath tumour was suggested due to the hypoechoic appearance with posterior acoustic enhancement on ultrasonography and location of the lesion in the region of facial region. The presence of intrinsic blood flow on colour doppler sonography and peripheral nerve continuity suggests the diagnosis of peripheral nerve sheath tumour. However the absence of blood flow on sonography in our case was against the diagnosis of peripheral nerve sheath tumour.

A USG guided FNAC was then performed which aspirated foul smelling pultacoeus cheese-like material and on cytological examination confirmed an epidermoid cyst (Figure 3). Surgical excision was done and histopathology confirmed the diagnosis of epidermoid cyst (Figure 4).

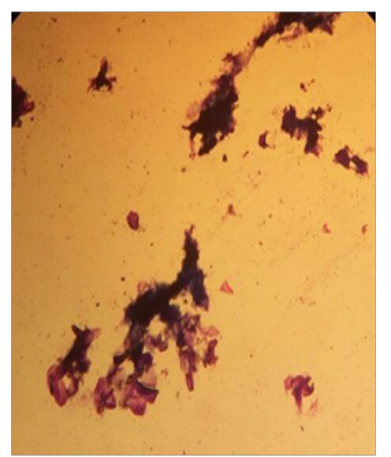

Figure 3 FNAC showing superficial squamous cells only

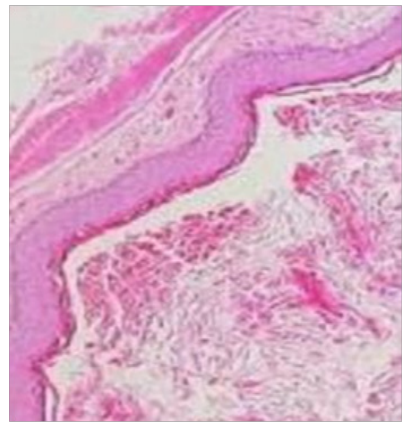

Figure 4 Histopathology showing keratin with stratified squamous epithelium with a granular layer.

\section{Discussion}

Epidermoid cysts also known as epidermal inclusion cysts, or sebaceous cysts (a misnomer) are the most common benign cutaneous cysts, and are most commonly located over head and neck and back. It results from implantation of epidermal elements in to the dermis with trauma being the most common cause. Other causes have been demonstrated to involve exposure to UV light, and human papilloma virus (HPV) infection. ${ }^{1,2}$ These are more commonly seen in males as compared to females. These cysts are usually asymptomatic but may become secondarily infected with chronic irritation and repetitive trauma resulting in pain, swelling and tenderness. The size of cysts varies from 0.5 to $4 \mathrm{~cm}$ in diameter. ${ }^{3}$ On ultrasound, epidermal inclusion cysts appear as well-circumscribed round or oval hypoechoic lesions with scattered echogenic reflectors and no evidence of internal blood flow on doppler. Yuan WH et al. ${ }^{4}$ in their clinicopathological and radiological study reviewed and analyzed the ultrasonographic features of both ruptured and unruptured epidermoid cysts. The diagnosis of each lesion was confirmed by histopathology. The authors reported that unruptured epidermoid cysts were noted to be oval with well-circumscribed boundaries, of oval shape with peripheral halo and lack of blood flow signals $(\mathrm{P}<.05)$. In contrast, ruptured epidermoid cysts usually had a lobulated shape, a slightly poorly defined boundary, the absence of a halo, and intermediate grades of vascularity. The other features such as size and dermal attachment, a hypoechoic echo texture, posterior acoustic enhancement, and the presence of intralesional hyperechoic strips and hypoechoic debris, showed no significant differences between the groups $(\mathrm{P}>.05){ }^{4}$

In addition to ultrasound, magnetic resonance imaging (MRI) has become a popular tool in the evaluation of epidermoid cysts which are located in the scrotal region. Due to the rare possibility of malignant transformation, Investigations such as FNAC, USG and MRI are indispensible in the diagnosis and evaluation of lesions with atypical presentation and unusual locations. The gold standard however remains surgical excision. The prognosis is very good with rare chances of relapse and malignancy. ${ }^{5,6}$ The diagnosis in our case was also confirmed with USG guided FNAC and histopathology after surgical excision.

Malignant peripheral nerve sheath tumours affect both male and female patients without significant sex predilection and usually occur in patients 20-50years of age. These patients clinically present with a soft-tissue mass involving major nerves such as sciatic nerve and causing pain or other neurological symptoms like muscle weakness and sensory deficits. The peripheral nerve sheath tumours (benign as well as malignant) on ultrasonography are often hypoechoic with posterior acoustic enhancement. The presence of intrinsic blood flow on colour doppler ultrasonography and peripheral nerve continuity suggests the diagnosis of a peripheral nerve sheath tumour. Ultrasonography cannot however reliably distinguish between benign and malignant nerve peripheral nerve sheath tumours. Lin et al. observed target appearance on ultrasonography (hyperechoic center with a hypoechoic periphery) in three of the 12 peripheral nerve sheath tumors, especially benign. The target appearance is believed to be due to a central fibrocollagenous and a peripheral myxomatous region. No malignant peripheral nerve sheath tumors showed a target appearance, which is similar to findings described with MRI. ${ }^{7}$

\section{Conclusion}

We hereby report this case due to atypical clinical features (size, colour and immobility) and doubtful ultrasound picture. Surgical excision remains the treatment of choice although investigations such as FNAC and ultrasonography are helpful especially in case of lesions located in the preauricular region. Complications are rare, but can include infection, scarring from excision and recurrence. Malignant transformation is very rare in epidermoid cysts.

\section{Acknowledgements}

None.

\section{Conflict of interest}

Author declares that there is no conflict of interest.

\section{References}

1. King ES. Post-traumatic epidermoid cysts of hands and fingers. $\mathrm{Br} J$ Surg. 1933;21:29-43.

2. Ettinger RL, Manderson RD. Implantation keratinizing epidermoid cysts: A review and case history. Oral Surg Oral Med Oral Pathol. 1973;36(2):225-230. 
3. Lee HS, Joo KB, Song HT, et al. Relationship between sonographic and pathologic findings in epidermal inclusion cysts. $J$ Clin Ultrasound. 2001;29(7):374-383.

4. Yuan WH, Hsu HC, Lai YC, et al. Differences in sonographic features of ruptured and unruptured epidermal cysts. J Ultrasound Med. 2012;31(2):265-272.

5. Zuber TJ. Minimal excision technique for epidermoid (sebaceous) cysts. Am Fam Physician. 2002;65(7):1409-1412.
6. Lopez-Rios F, Rodriguez-Peralto JL, Castano E, et al. Squamous cell carcinoma arising in a cutaneous epidermal cyst: case report and literature review. Am J Dermatopathol. 1999;21(2):174-177.

7. David L, Reynolds, Jon A, et al. Sonographic characteristics of peripheral nerve sheath tumours. American Journal of Roentgenology. 2004;182(3):741-744 